\title{
3D Shape Classification Using Commute Time
}

\author{
Muhammad Haseeb and Edwin R. Hancock ${ }^{\star}$ \\ Department of Computer Science, The University of York, UK
}

\begin{abstract}
This paper describes a commute-time based 3D shape descriptor that is robust with respect to changes in pose and topology. A new and completely unsupervised mesh segmentation algorithm is proposed, which is based on the commute time embedding of the mesh and the k-means clustering using the embedded mesh vertices. We use the discrete Laplace-Beltrami operator to construct the graph Laplacian.
\end{abstract}

Keywords: 3D Mesh Clustering, Commute Time Embedding, Shape Descriptor.

\section{Introduction}

Despite significant efforts in the past 10 to 15 years, graph clustering and classification remain an open challenge in the machine learning community. One of the most promising approaches is to use spectral clustering methods which exploits graph representations of the data and locate clusters by partitioning the graph that optimize an edge cut criterion. Early spectral approaches recursively compute the normalized cut [1] over the graph using the first non-zero Laplacian eigenvector (also known as the Fiedler vector [2] and are referred to as spectral bi-partitioning (SB) methods. Unfortunately, this does not guarantee good clusters as the normalized cut is computed recursively irrespective of the global structure of the data [3]. Qiu and Hancock [4] have used commute time for the purpose of image segmentation and show that the commute time method outperforms the normalized cut.

Recently, the graph spectral methods defined in the context of clustering have been applied to 3D shape processing. Here the discrete representation of 3D shape in the computer is a mesh, or sometimes a point set. In this context, spectral invariants such as the eigenfunctions of the Laplacian operator can be used for near-isometric shape matching. For instance, Mateus et al. [5] used eigenmaps obtained by the first $k$ eigenfunctions of the Laplace operator as low-dimensional Euclidean representations of nonrigid shapes for the purpose of 3D point registration. Cuzzolin et al. [6] and Yamasaki et al. [7] have performed segmentation for mesh sequences. However, the former method computes only protrusions, while the latter uses an additional skeleton. In [6], the authors use locally linear embedding (LLE) to represent a cloud of points and perform segmentation in the LLE space. The segments obtained are then propagated across time to obtain a temporally coherent segmentation of a voxel-sequence into protrusions of the shape. The method works well for rigid body parts (such as head, hands and legs etc), but cannot be used directly for identifying rigid body-parts (for example, separating the upper-arm from the lower-arm).

\footnotetext{
^ Edwin R. Hancock is supported by a Royal Society Wolfson Research Merit Award.
} 
Spectral methods can also be used to measure the similarity of 3D shapes. For instance, diffusion geometry methods were used to define low dimensional representations for manifolds. Rustamov [8] has suggested using the eigendecomposition of the Laplace-Beltrami operator to construct an isometric invariant surface representation, aiming to measure similarity between non-rigid shapes, rather than for correspondence detection. The Global Point Signature (GPS) suggested by Rustamov [8] for shape comparison employs the discrete Laplace-Beltrami operator, which globally captures the shapes geometry. The Laplace-Beltrami operator was later employed by many other researchers. For instance, Sun et al. [9] defined a point signature based on the properties of the heat diffusion process on a shape, referred to as the Heat Kernel Signature (HKS). HKS is obtained by restricting the well-known heat kernel to the temporal domain. Ovsjanikov et al. [10] employed a heat diffusion process to construct the Heat Kernel Maps for the shape matching. Castellani et al. [11] have extended Heat Kernel Signature (HKS). The local heat kernel values observed at each point are accumulated into a histogram for a fixed number of scales leading to the so-called Global Heat Kernel Signature (GHKS).

In this paper we construct a novel 3D shape distribution for the purpose of 3D object classification. The method commence from a modification of the $3 \mathrm{D}$ shape distribution reported in [12]. Firstly, instead of using Euclidean distances between pair of points on the shape, we use commute time distance computed from the eigenvalues and the eigenfunctions of the Laplace-Beltrami operator. Secondly we put a restriction on the pair of points being selected more than once. The empirical results show that the distribution computed using our method gives a better shape signature than [12] and [8].

\section{Commmute Time}

In this section, we briefly review how to compute the commute time and describe the relationships to the graph Laplacian. Commute time is the time taken by a random walker on a graph walking from a node $u$ to node $v$ and then back to node $u$. The commute time can be computed from the Laplacian spectrum as it has a close relationship with the graph Laplacian and heat kernel.

Consider a weighted graph by the triple $\Gamma=(V, E, \Omega)$, where $V$ is the set of nodes, $E \subseteq V \times V$ is the set of edges, and $\Omega$ is the weighted adjacency matrix.

$$
\Omega(u, v)= \begin{cases}w(u, v) & \text { if }(u, v) \in E \\ 0 & \text { otherwise }\end{cases}
$$

where $w(u, v)$ is the weight on the edge $(u, v) \in E$. Furthermore, let $T=\operatorname{diag}\left(d_{u} ; u \in\right.$ $V)$ be the diagonal weighted degree matrix with elements given by the degrees of the nodes, $d_{u}=\sum_{v=1}^{|V|} w(u, v)$. The unnormalized weighted Laplacian matrix is given by $L=T-\Omega$ and the normalized weighted Laplacian matrix is defined to be $\mathcal{L}=$ $T^{-1 / 2} L T^{-1 / 2}$ and has elements

$$
\mathcal{L}(u, v)= \begin{cases}1 & \text { if } u=v \\ -\frac{w(u, v)}{\sqrt{d_{u} d_{v}}} & \text { if } u \neq v \text { and }(u, v) \in E \\ 0 & \text { otherwise }\end{cases}
$$


The spectral decomposition of the normalized Laplacian is $\mathcal{L}=\Phi \Lambda \Phi^{T}$ where $\Lambda=$ $\operatorname{diag}\left(\lambda_{1}, \lambda_{2}, \ldots, \lambda_{|V|}\right)$ is the diagonal matrix with the ordered eigenvalues as the elements satisfying the condition $0=\lambda_{1} \leq \lambda_{2} \leq \ldots, \leq \lambda_{|V|}$ and $\Phi=\left(\phi_{1}\left|\phi_{2}\right| \ldots \mid \phi_{|V|}\right)$ is the matrix with the ordered eigenvectors as columns.

The hitting time $O(u, v)$ of a random walk on a graph is defined as the expected number of steps before node $v$ is visited, commencing from node $u$. The commute time $C T(u, v)$, on the other hand, is the expected time for the random walk to travel from node $u$ to reach node $v$ and then return. As a result $C T(u, v)=O(u, v)+O(v, u)$. In terms of the eigenvectors of the normalized Laplacian the commute time matrix is given by

$$
C T(u, v)=\operatorname{vol} \sum_{i=2}^{|V|} \frac{1}{\lambda_{i}}\left(\frac{\phi_{i}(u)}{\sqrt{d_{u}}}-\frac{\phi_{i}(v)}{\sqrt{d_{v}}}\right)^{2}
$$

where $v o l=\sum_{v \in V} d_{v}$ is the volume of the graph.

The commute time embedding is a mapping from the data space into a Hilbert subspace, which preserves the original commutes times. It has some properties similar to existing embedding methods including principal component analysis [13] (PCA), the Laplacian eigenmap [3] and the diffusion map [14]. The embedding of the nodes of the graph into a vector space that preserves commute time has the co-ordinate matrix

$$
\Theta=\sqrt{\operatorname{vol}} \Lambda^{-1 / 2} \Phi^{T} T^{-1 / 2}
$$

The columns of the matrix are vectors of embedding co-ordinates for the nodes of the graph.

\section{Laplace-Beltrami Operator}

Let $f$ be a real valued funtion defined on a differentiable manifold $\mathcal{M}$ with Riemannian metric. The LaplaceBeltrami operator, like the Laplacian, is the divergence of the gradient of $f$ i.e.

$$
\Delta f=\operatorname{div}(\operatorname{grad}(f))
$$

where grad and div are the gradient and divergence on the manifold respectively. The Laplace-Beltrami operator is a semi-positive definite operator. Most of the techniques [8] [15] for characterizing points on non rigid 3D shapes use the eingenpairs of the Laplace-Beltrami operator. The combinatorial Laplacian is suitable for the meshes and its does not contain much information about the shape. The discrete Laplacian or Laplace-Beltrami operator captures the geometric and topological properties of the surface. There are many schemes proposed to construct the discrete Laplacian that estimates the Laplace-Beltrami operator. Majority of them use the method of cotagents. However, the method described by Destrun et al. [16] and Meyer at al. [15] are more stable than the others. Xu [17] modified the method proposed by Meyer et al. This modification gives better convergence properties. In this paper we will follow Xu's method to construct the discrete Laplacian (Laplace-Beltrami operator). 


\subsection{The Generalized Eigenvalue Problem}

For a function $f$ defined on the surface, the Laplacian $\Delta f$ is approximated as

$$
\Delta f \approx \frac{1}{s_{i}} \sum_{j \in N(i)} w_{i j}\left[f\left(p_{j}\right)-f\left(p_{i}\right)\right]
$$

where $N(i)$ are the neighbors for the vertex $p_{i}$ and $w_{i j}$ is the weight assigned to the edge between point $p_{i}$ and $p_{j}$. The above formula can be written as $\Delta f \approx L f$. Here $L$ is the discrete Laplacian matrix. The weight $w_{i j}$ of the edge is given by

$$
w_{i, j}=\frac{\cot \alpha_{i j}+\cot \beta_{i j}}{2}
$$

The angles appearing in this formula i.e. $\alpha_{i j}$ and $\beta_{i j}$ are shown in the figure 1 . The area $s_{i}$ is also shown as the shaded region in the same figure. We compute the Laplacian, which has the entries as follows

$$
L(i, j)= \begin{cases}\sum_{k} w(i, k) / s_{i} & \text { if } i=j \\ -w(i, j) / s_{i} & \text { if } i \text { and } j \text { are adjacent } \\ 0 & \text { otherwise }\end{cases}
$$

The standard eigenvalue problem for $L$ is $L \phi=\lambda \phi$, where $\lambda$ is the eigenvalue of $L$ and $\phi$ is the corresponding eigenvector. The area $s_{i}$ at each vertex is computed as

$$
s_{i}=\frac{\cot \alpha_{i j}+\cot \beta_{i j}}{8}\left\|p_{i}-p_{j}\right\|^{2}
$$

Since the areas $s_{i}$ computed at the vertices of the mesh are different, hence, the discrete Laplacian matrix $L$ computed is not symmetric. This may cause the eigenvalues and eigenfunction to be complex. Therefore, we solve the generalized eigenvalue problem. Let $S$ be the diagonal matrix with entries $S_{i i}=s_{i}$ and $W_{i j}=w_{i j}$ be the symmetric weight matrix. Since $L=S^{-1} W$, therefore, we can rewrite the equation $L \phi=\lambda \phi$ as $S^{-1} W \phi=\lambda \phi$ or

$$
W \phi=\lambda S \phi
$$

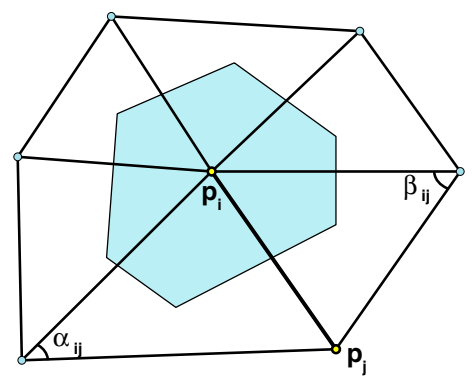

Fig. 1. Definitions of the angles and the area appearing in the discrete Laplace-Beltrami operator 
Once we have the eigenvalues and eigenfunction of $L$ to hand, we can compute the commute time matrix using the equation 1 Where we replace the degree of the nodes (i.e. $d_{u}$ and $d_{v}$ ) by the area associated with the vertices (i.e. $s_{i}$. and $s_{j}$ respectively). We replace the $v o l$ in the original equation by $\sum_{i} s_{i}$.

\section{Shape Classification Using Commute Time}

The commute time embedding gives a deformation independent embedding of a 3D shape into a high dimensional space. In this paper, we compute a shape descriptor from the commute time embedding. We use Laplace-Beltrami operator detailed in Section 3 to estimate the Laplacian of the shape. From the eigenvalues and eigenvectors of the Laplacian obtained, we compute the commute time matrix using the procedure given in Section 2. We use a modification of $D 2$ distributions introduced in [12]. D2 distribution is essentially, the histogram of pairwise Euclidean distance between the points uniformly sampled from the surface. To compute our new shape descriptor, we use the commute time distance instead of the Euclidean distance. We also restrict a pair of point from being sampled more than once.

\section{Experimental Results}

In this section, we provide some experimental investigations. We focus on the use of commute time embedding of 3D shapes in two different settings. The first is an investigation of using the the commute time embedding for the purpose of partitioning the 3D shape into its parts. The second investigation is about using the modified shape distribution of Osada et al [12] computed by employing the commute time distance instead of the Euclidean distance.

In our first experiment we use the commute time embedding coordinates computed using equation 2 to partition six deformations of a human body selected from the Nonrigid world 3D database [18] shown in figure 2] The database contains a total of 148 objects, including 9 cats, 11 dogs, 3 wolves, 17 horses, 15 lions, 21 gorillas, 1 shark, 24 female figures, and two different male figures, containing 15 and 20 poses. The database also contains 6 centaurs, and 6 seahorses for partial similarity experiments.
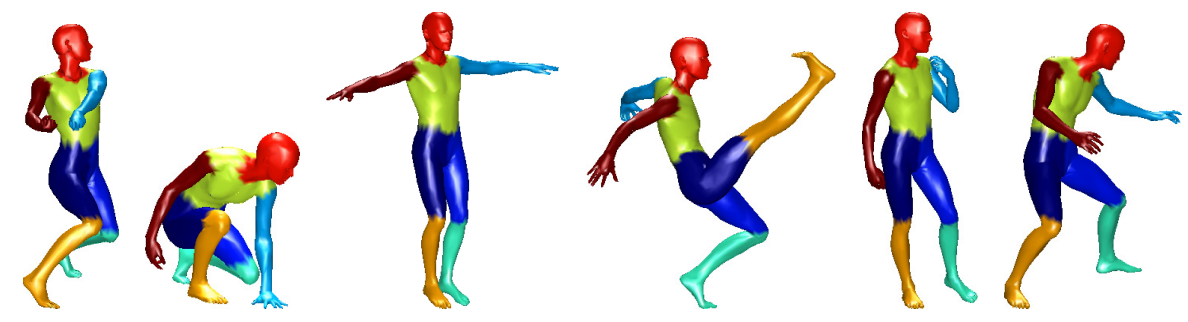

Fig. 2. The k-means clustering on the Commute Time coordinates results in segmentation of six deformations of a 3D shape 


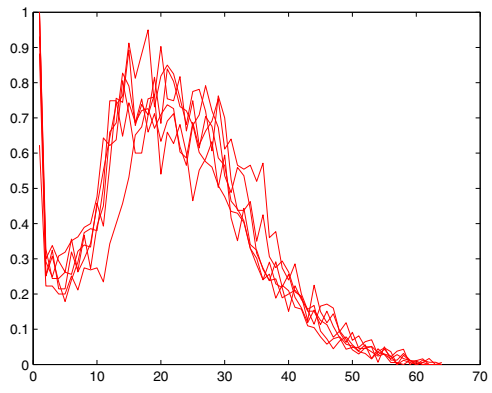

(a)

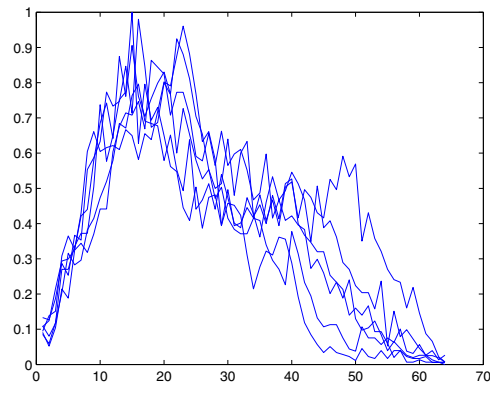

(b)

Fig. 3. The histogram for the six 3D shapes shown in figure 2. a) The commute time histogram b) The Euclidean histogram.

Each object contains approximately 3500 vertices. Figure 2 shows the result of the 3D shape, pose invariant segmentation using the k-means clustering on the commute time coordinates.

In the second experiment, we construct the shape distribution for different six different deformations of each of the three 3D shapes shown in figure 4(a) Figure 3(a) shows the shape descriptors for the six deformations using commute times. The shape descriptors for the same six deformations using Euclidean distances are shown in figure 3(b) This shows that the shape descriptor computed using commute time is more robust to shape deformations. We find the distance between each pair of the distributions using Bhattacharyya distance [19]. We project the distance matrix into vector space using classical multi-dimensional scaling (MDS). Figure 4 shows that the commute time shape distribution clusters similar shapes better than the Euclidean shape distribution.

For the final experiment we use the Watertight Benchmark which contains 400 closed surface shapes, grouped into 20 classes with 20 shapes each. We query every shape in the benchmark against all the other shapes. We compute three retrieval statics i.e. nearest first tier (FT), second tier (ST) and nearest neighbor (NN) for Osada's D2, Rastamov's GPS, Rastamov's Volumetric Shape Descripor (VSD) and the commute time. The shape retrieval results of the experiment are summarized in the table 1 which suggest that the commute time gives a better shape signature.

Table 1. Shape retrieval statistics

\begin{tabular}{|l|c|c|c|}
\hline Descriptor & FT & ST & NN \\
\hline Osada's D2 & $49.2 \%$ & $67.8 \%$ & $71.5 \%$ \\
\hline Rustamov's GPS & $46.3 \%$ & $58.0 \%$ & $79.1 \%$ \\
\hline Rustamov's VSD [20] & $48.7 \%$ & $62.0 \%$ & $81.3 \%$ \\
\hline Commute Time & $49.7 \%$ & $69.2 \%$ & $86.7 \%$ \\
\hline
\end{tabular}




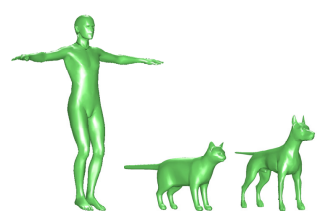

(a)

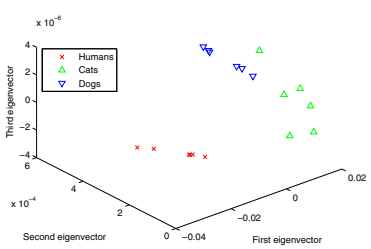

(b)

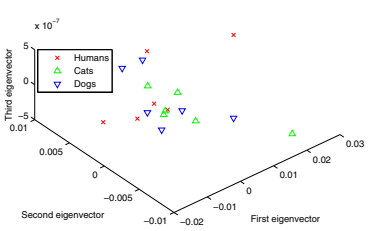

(c)

Fig. 4. a) Three shapes used in clustering experiment (six deformations of each shape are used). b) The classical MDS projection of the shape similarities as computed using the commute time distributions. c) The classical MDS projection of the shape similarities as computed using the D2 distributions.

\section{Conclusions}

In this paper we have investigated how the commute time between the vertices on mesh can be used to partition the 3D shape. We also used commute time distance to construct the 3D shape distribution for the purpose of 3D shape clustering and 3D shape classification. The empirical results show that commute time is a better choice for shape classification problem. In future we would like to extend our shape descriptor by employing the geodesic distances between each pair of vertices.

Acknowledgement. Edwin R. Hancock was supported by a Royal Society Wolfson Research Merit Award.

\section{References}

1. Shi, J., Malik, J.: Normalized cuts and image segmentation. IEEE Transactions on Pattern Analysis and Machine Intelligence 22, 888-905 (2000)

2. Chung, F.R.K.: Spectral Graph Theory. American Mathematical Society (1997)

3. Belkin, M., Niyogi, P.: Laplacian eigenmaps for dimensionality reduction and data representation. Neural Computation 15, 1373-1396 (2003)

4. Qiu, H., Hancock, E.R.: Clustering and embedding using commute times. IEEE Trans. Pattern Anal. Mach. Intell. 29, 1873-1890 (2007)

5. Mateus, D., Horaud, R., Knossow, D., Cuzzolin, F., Boyer, E.: Articulated shape matching using laplacian eigenfunctions and unsupervised point registration. In: CVPR (2008)

6. Cuzzolin, F., Mateus, D., Knossow, D., Boyer, E., Horaud, R.: Coherent laplacian 3-D protrusion segmentation. In: CVPR (2008)

7. Lee, N.S., Yamasaki, T., Aizawa, K.: Hierarchical mesh decomposition and motion tracking for time-varying-meshes. In: ICME, pp. 1565-1568. IEEE (2008)

8. Rustamov, R.M.: Laplace-beltrami eigenfunctions for deformation invariant shape representation. In: Symposium on Geometry Processing, pp. 225-233 (2007)

9. Sun, J., Ovsjanikov, M., Guibas, L.: A concise and provably informative multi-scale signature based on heat diffusion. In: Proceedings of the Symposium on Geometry Processing, SGP 2009, pp. 1383-1392. Eurographics Association, Aire-la-Ville (2009) 
10. Ovsjanikov, M., Mérigot, Q., Mémoli, F., Guibas, L.J.: One point isometric matching with the heat kernel. Comput. Graph. Forum 29, 1555-1564 (2010)

11. Castellani, U., Mirtuono, P., Murino, V., Bellani, M., Rambaldelli, G., Tansella, M., Brambilla, P.: A New Shape Diffusion Descriptor for Brain Classification. In: Fichtinger, G., Martel, A., Peters, T. (eds.) MICCAI 2011, Part II. LNCS, vol. 6892, pp. 426-433. Springer, Heidelberg (2011)

12. Osada, R., Funkhouser, T., Chazelle, B., Dobkin, D.: Matching 3d models with shape distributions. In: Proceedings of the International Conference on Shape Modeling \& Applications, SMI 2001, pp. 154-167. IEEE Computer Society, Washington, DC (2001)

13. Jolliffe, I.: Principal Component Analysis, 2nd edn. American Mathematical Society. Springer-Verlag New York, Inc., New York (2002)

14. Lafon, S., Lee, A.: Diffusion maps: a unified framework for dimension reduction, data partitioning and graph subsampling. IEEE Transactions on Pattern Analysis and Machine Intelligence (2005)

15. Meyer, M., Desbrun, M., Schröder, P., Barr, A.H.: Discrete Differential-Geometry Operators for Triangulated 2-Manifolds. In: Hege, H.C., Polthier, K. (eds.) Visualization and Mathematics III, pp. 35-57. Springer, Heidelberg (2003)

16. Desbrun, M., Meyer, M., Schröder, P., Barr, A.H.: Implicit fairing of irregular meshes using diffusion and curvature flow. In: Proceedings of the 26th Annual Conference on Computer Graphics and Interactive Techniques, SIGGRAPH 1999, pp. 317-324. ACM Press/AddisonWesley Publishing Co., New York (1999)

17. $\mathrm{Xu}, \mathrm{G} .:$ Discrete laplace-beltrami operator on sphere and optimal spherical triangulations. Int. J. Comput. Geometry Appl. 16, 75-93 (2006)

18. Alexander, Bronstein, M.: Nonrigid world 3d database v $1.0 @$ ONLINE (2009)

19. Bhattacharyya, A.: On a measure of divergence between two statistical populations defined by their probability distributions. Bulletin of the Calcutta Mathematics 35, 99-110 (1943)

20. Rustamov, R.M.: Robust volumetric shape descriptor. In: Eurographics Workshop on 3D Object Retrieval, pp. 1-5 (2010) 